\title{
LIIDIA KIISK DEFENDED HER DOCTORAL THESIS
}

\author{
Andres Arend \\ Department of Anatomy, University of Tartu, Tartu, Estonia
}

On January 27, 2017 Liidia Kiisk successfully defended her doctoral thesis entitled Long-term nutritional study: anthropometrical and clinico-laboratory assessments in renal replacement therapy patients after intensive nutritional counselling and received the degree of Doctor of Philosophy (Medicine) from the Council of the Faculty of Medicine of the University of Tartu, Estonia. The dissertation was supervised by Professor Mai Rosenberg from the Department of the Internal Medicine of the Institute of Clinical Medicine, University of Tartu, and by Professor Emeritus Helje Kaarma from the Centre for Physical Anthropology, University of Tartu. The opponent was Professor Inga Arune Bumblyte from the Faculty of Medicine of the Lithuanian University of Health Sciences.

In her dissertation, Liidia Kiisk has performed a very extensive and complex study of the body composition characteristics and nutritional habits of the end-stage chronic kidney disease patients. One of the important aims of the study was to evaluate if intensive nutritional counselling has an effect on the anthropometrical, biochemical and nutritional parameters of kidney transplant patients. The result of the long-term follow-up of kidney transplant patients demonstrated clearly that intensive nutritional counselling was effective to avoid body weight gain, which is considered an important risk factor for the development of chronic transplant nephropathy. Furthermore, the effect of intensive nutritional counselling on the changes of biochemical parameters revealed the normalization of the inflammatory status in most patients. The associations between anthropometrical and biochemical parameters were, however, found to differ in male and female patients. If in male patients several anthropometrical parameters were associated with C-reactive protein, then in females with lipids. These associations deserve special attention as inflammation and hyperlipidaemia are well-known cardiovascular risk factors.

It has to be emphasized that the dissertation of Liidia Kiisk has obvious practical value which exceeds the limits of the original dissertation. The author of the dissertation, who is currently working as a dietitian at Tartu University 
Hospital, has drawn up a systematized list of diets and compiled guidebooks which follow the contemporary principles of feeding treatments. By today, based on the initiative of Liidia Kiisk, a common system of diets for treatment has been implemented not only at Tartu University Hospital but also in all the hospitals and health care centres in Estonia. 